\title{
Thirty-Six-Month Amyloid Positron Emission Tomography Results Show Continued Reduction in Amyloid Burden with Subcutaneous Gantenerumab
}

\author{
G. Klein ${ }^{1}$, P. Delmar ${ }^{1}$, G.A. Kerchner ${ }^{1}$, C. Hofmann ${ }^{1}$, D. Abi-Saab ${ }^{1}$, A. Davis ${ }^{2}$, N. Voyle ${ }^{2}$, M. Baudler ${ }^{1,3}$, \\ P. Fontoura ${ }^{1}$, R. Doody ${ }^{1,3}$
}

1. F. Hoffmann-La Roche Ltd, Basel, Switzerland; 2. Roche Products Ltd, Welwyn Garden City, UK; 3. Genentech Inc., South San Francisco, CA, USA.

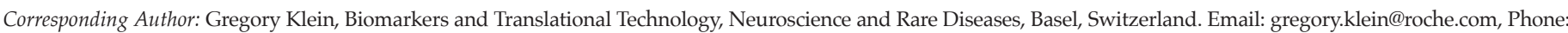
(+41) 616820759

J Prev Alz Dis 2021;1(8):3-6

Published online December 3, 2020, http://dx.doi.org/10.14283/jpad.2020.68

\begin{abstract}
Previous findings from the positron emission tomography (PET) substudy of the SCarlet RoAD and Marguerite RoAD open-label extension (OLE) showed gantenerumab doses up to 1200 mg every 4 weeks administered subcutaneously resulted in robust beta-amyloid $(A \beta)$ plaque removal over 24 months in people with prodromal-to-moderate Alzheimer's disease (AD). In this 36-month update, we demonstrate continued reduction, with mean (standard error) centiloid values at 36 months of -4.3 (7.5), 0.8 (6.7), and 4.7 (8.0) in the SCarlet RoAD (double-blind pooled placebo and active groups), Marguerite RoAD double-blind placebo, and Marguerite RoAD double-blind active groups respectively, representing a change of -57.0 (10.3), -90.3 (9.0), and -74.9 (10.5) centiloids respectively. These results demonstrate that prolonged gantenerumab treatment, at doses up to $1200 \mathrm{mg}$, reduces amyloid plaque levels below the amyloid positivity threshold. The ongoing GRADUATE Phase III trials will evaluate potential clinical benefits associated with gantenerumab-induced amyloidlowering in people with early (prodromal-to-mild) AD.
\end{abstract}

Key words: Gantenerumab, Alzheimer's disease, positron emission tomography, amyloid.

\section{Introduction}

A lzheimer's disease (AD) accounts for $60-80 \%$ of all cases of dementia globally (1). Currently, the available treatments for $\mathrm{AD}$ offer only limited benefits and there is an urgent need for diseasemodifying therapies that reverse neuropathologic changes, or slow or stop neurodegeneration $(1,2)$.

$A D$ pathogenesis is driven by the gradual accumulation of beta-amyloid $(A \beta)$ plaques and neurofibrillary tangles (NFTs) in the brain $(1,3)$. In vitro and in vivo evidence suggests that soluble $A \beta$ oligomers and insoluble $A \beta$ plaques contribute to cognitive failure by causing neuronal loss, synaptic dysfunction and disconnection syndromes $(4,5)$. The recognition of $A \beta$ accumulation as the earliest identifiable marker of $\mathrm{AD}$

has led to the development of amyloid positron emission tomography (PET), a neuroimaging technique that can be utilized to visualize $A \beta$ accumulation that helps improve diagnostic accuracy and may also facilitate appropriate participant selection in clinical trials (6).

Gantenerumab is a fully humanized, anti-A $\beta$ immunoglobulin (Ig) G1 that binds to $A \beta$ species with high affinity for aggregated forms, including oligomers and plaques, and is thought to remove $A \beta$ via microglia-mediated phagocytosis $(7,8)$. The longterm, pharmacodynamic effect of gantenerumab-induced $A \beta$ plaque removal in participants with prodromalto-mild and mild-to-moderate AD is currently being investigated in the PET substudies of the Phase III SCarlet RoAD (SR; NCT01224106) and Marguerite RoAD (MR; NCT02051608) open-label extension (OLE) studies, respectively (7). Interim results showed robust A $\beta$ plaque removal with gantenerumab doses up to $1200 \mathrm{mg}$ administered subcutaneously, with mean amyloid reductions of 59 centiloids and $51 \%$ of participants below the $\mathrm{A} \beta$ positivity threshold after 24 months (7). Here, we tested whether amyloid signal plateaus or continues to decline with continued therapy in the 36-month results of the ongoing OLE PET substudy.

\section{Methods}

\section{Participants and study design}

Complete details of the study designs and methodologies of SR and MR, the associated OLE studies, and the OLE PET substudies have been previously reported (7-9). Briefly, participants in the SR trial who received double-blind treatment and had $\geq 1$ follow-up visit and those who were currently enrolled in the MR trial were eligible for participation in the OLE. Various titration schemes were used to allow OLE participants to gradually reach the target dose of gantenerumab $1200 \mathrm{mg}$ per month while decreasing the risk of amyloid-related imaging abnormality (ARIA)-related adverse events. The target gantenerumab dose was reached within 6 to 10 
Table 1. Baseline characteristics of participants enrolled in the SR, MR-DBP, and MR-DBA cohorts, including 36-month completers

\begin{tabular}{|c|c|c|c|c|c|c|}
\hline \multirow[b]{2}{*}{ Characteristic } & \multicolumn{3}{|c|}{ Total study } & \multicolumn{3}{|c|}{ 36-month completers } \\
\hline & SR & MR-DBP & MR-DBA & SR & MR-DBP & MR-DBA \\
\hline Participants with at least 1 post-baseline scan, $n$ & 19 & 27 & 21 & 10 & 12 & 8 \\
\hline Age at start of OLE, median (IQR), years & $72(70-78)$ & $74(69-77)$ & $65(59-79)$ & $72(70-81)$ & $72(68-75)$ & $71(62-82)$ \\
\hline APOE $\varepsilon 4$ carriers, n $(\%)$ & $17(89)$ & $18(67)$ & $11(52)$ & $10(100)$ & $8(67)$ & $5(63)$ \\
\hline MMSE score at OLE baseline, mean (SD) & $19(4.8)$ & $22(4.5)$ & $19(5.0)$ & $19(4.0)$ & $21(5.0)$ & $23(3.4)$ \\
\hline OLE baseline amyloid burden, Centiloids, mean (SE) & $49.6(12.1)$ & $91.1(9.0)$ & $79.6(11.3)$ & $62.9(11.1)$ & $83.1(17.8)$ & 88.5 (20.5) \\
\hline
\end{tabular}

APOEع4, Apolipoprotein E; IQR, Interquartile range; MMSE, Mini-Mental State Examination; MR-DBA, Marguerite RoAD double-blind active; MR-DBP,@@@@@@@ Marguerite RoAD double-blind placebo; OLE, open-label extension; SE, standard error; SD, standard deviation; SR, SCarlet RoAD.

months for SR OLE participants, and 2 to 6 months in MR OLE participants.

Participants of the OLE substudy were divided into three cohorts based on their prior exposure to gantenerumab and their stage of AD. The SR cohort included SR participants with all SR treatment arms pooled together (received gantenerumab $105 \mathrm{mg}$ or $225 \mathrm{mg}$ or placebo every 4 weeks during the doubleblind phase), all participants in the SR cohort were off treatment for 16 to 19 months prior to OLE higher dosing. The MR double-blind placebo cohort (MR-DBP) included participants in the MR trial who received placebo during the double-blind phase and the MR double-blind active cohort (MR-DBA) included participants of the MR trial who received either 105 or $225 \mathrm{mg}$ gantenerumab during the double-blind phase.

\section{Amyloid- $\beta$ plaque PET imaging and quantification}

Amyloid PET scans were obtained at baseline and at 12 , 24, and 36 months after baseline using intravenous $370 \mathrm{MBq}{ }^{18} \mathrm{~F}$-florbetapir, with each 15 -minute scan obtained 50 minutes after ${ }^{18} \mathrm{~F}$-florbetapir injection. Participants who received a PET scan during the doubleblind phase, within 9 to 12 months of OLE dosing, were not scanned at OLE baseline to minimize participant burden.

Volume-weighted, gray matter-masked standard uptake value ratios (SUVR) were calculated for six bilateral cortical regions using the Automated Anatomical Labeling (AAL) template, normalized by a cerebellar cortex reference region $(10,11)$. SUVR values were then converted to centiloid values as previously described, using the following linear transformation: Centiloid = SUVR*184.12 - $233.72(7,12)$. The threshold for amyloid positivity has been previously established as 24 centiloids, which corresponds to 1.40 SUVR units. The amyloid positivity threshold represents the quantitative threshold that best discriminates pathologically verified absence of plaques or sparse plaques from moderate-tofrequent plaques (13).

\section{Statistical analysis}

This analysis included all study participants who had a PET scan at OLE baseline (or 9-12 months prior to OLE dosing) and received $\geq 1$ follow-up scan. PET centiloid values were analyzed using a mixed model for repeated measures (MMRM), with treatment visit, treatment group, and the interaction for treatment group by visit as independent variables. An unstructured covariance matrix was used to capture within-participant correlation.

\section{Results}

\section{Participant characteristics}

A total of 67 participants with at least 1 post-baseline scan were enrolled in the OLE PET substudy (SR, $\mathrm{n}=19$; MR-DBP, $\mathrm{n}=27$; MR-DBA, $\mathrm{n}=21$ ). A total of 30 participants completed the 36-month scan (SR, $\mathrm{n}=10$; MR-DBP, $\mathrm{n}=12$; MR-DBA, $\mathrm{n}=8$ ). The baseline characteristics for both the overall population and the 36-month completers are shown in Table 1. More than half of the participants in each cohort were Apolipoprotein E (APOE) $\varepsilon 4$ carriers (SR, 89\%; MR-DBP, 67\%; MR-DBA, 52\%). Across all three cohorts, the mean [SE] baseline amyloid burden in centiloids was above the positivity threshold (SR, 49.6 [12.1]; MR-DBP, 91.1 [9.6]; MR-DBA, 79.6 [10.9]).

\section{Amyloid PET results}

Consistent with our previous report, reductions in mean amyloid burden were observed across cohorts after 12 and 24 months of open-label therapy, with 37\% and $52 \%$ of participants, respectively, reaching levels below the amyloid positivity threshold (Figure 1) (7). Continued reductions beyond 24 months were observed after 36 months, with mean amyloid levels approaching zero centiloids across all cohorts. The absolute mean (SE) amyloid burden after 36 months were -4.3 (7.5), 0.8 (6.7), and 4.7 (8.0) centiloids for the SR, MR-DBP and 
Figure 1. Reduction of amyloid burden towards zero centiloids after 36 months of open-label therapy

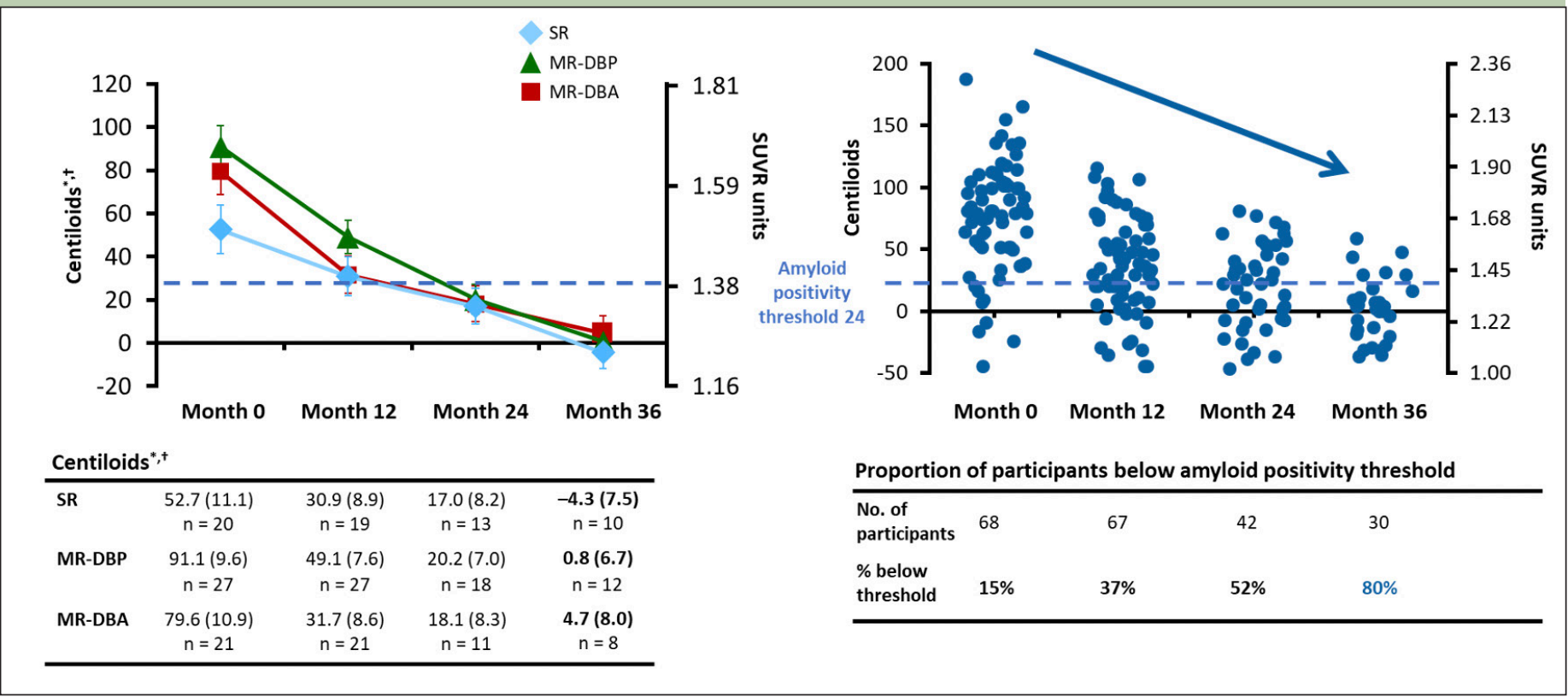

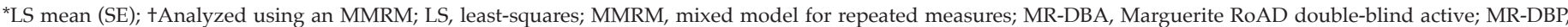
Marguerite RoAD double-blind placebo; SE, standard error; SR, SCarlet RoAD; SUVR, standard uptake value ratio.

MR-DBA cohorts, respectively, representing a change of $-57.0(10.3),-90.3(9.0),-74.9$ (10.5) centiloids respectively. Furthermore, the proportion of participants below the amyloid positivity threshold was 24 of 30 participants (80\%) at 36 months (Figure 1).

\section{Discussion}

This 36-month OLE PET substudy investigated the effect of gantenerumab on $A \beta$ plaque removal on participants with prodromal-to-moderate AD. Prior results have shown that while the three cohorts began with considerably different mean baseline centiloid values, all three cohorts demonstrated a mean centiloid value just below the amyloid positivity threshold after 24 months of treatment with gantenerumab $1200 \mathrm{mg}$ every 4 weeks. The latest results showed continued $\mathrm{A} \beta$ reduction with gantenerumab treatment below the amyloid positivity threshold, without plateau, with $80 \%$ of completers below the amyloid positivity threshold after 36 months of open-label therapy. Mean centiloid values of all three cohorts at this time are near a value of zero, which represents the mean amyloid burden expected in a healthy control group (12). Given that the SR and MR-DBA groups may have experienced some amyloid reduction due to low-dose gantenerumab treatment during the double-blind period of the SR and MR studies, the 90-centiloid reduction seen in the MR-DBP group represents the amyloid reduction that could be expected in a treatment-naïve population. The consistent reduction in $A \beta$ suggests that gantenerumab is able to remove $A \beta$ species successfully.

These findings may translate to clinical benefit in people with prodromal-to-mild $\mathrm{AD}$ as other studies with aducanumab and lecanemab (BAN2401) have observed amyloid PET reduction as well as clinical efficacy $(7,14,15)$. Specifically, in a Phase Ib placebocontrolled study, aducanumab demonstrated reduced brain amyloid plaque levels after 24 months with a reduction in clinical decline as measured by the Clinical Dementia Rating-Sum of Boxes (CDR-SB) and MiniMental State Examination (MMSE) (14). In a Phase II placebo-controlled study, lecanemab produced a dosedependent reduction in amyloid plaque levels after 18 months and a reduction in clinical decline as measured by AD Composite Score (15). In light of these studies, the current PET results suggest that the process of $A \beta$ reduction at the gantenerumab dose of $1200 \mathrm{mg}$ every 4 weeks has the potential to produce clinical benefits. The precise relation between amyloid reduction and clinical benefit is still an open question, including the question of whether a reduction to below amyloid positivity or to centiloid zero makes a difference in the clinical outcome and management of patients with early AD. The ongoing GRADUATE Phase III program evaluates the safety and efficacy of gantenerumab, subcutaneously administered, in participants with early AD. This program includes two global, double-blind, placebo-controlled trials in people with early $\mathrm{AD}$, designed to maximize exposure to gantenerumab and to prospectively examine the correlation between amyloid-lowering and clinical outcomes.

Funding: This study was sponsored by F. Hoffmann-La Roche Ltd, Basel, Switzerland. 
Acknowledgments: We would like to thank all the participants and their families, the investigators and site staff, and the entire study team for their time and commitment to the SCarlet RoAD and Marguerite RoAD OLE studies. Medical writing support was provided by Joshua Quartey, BSc, of Health Interactions and was funded by F. Hoffmann-La Roche Ltd.

Conflict of interest disclosures: GK, PD, GAK, CH, DA-S and PF were full-time employees of F. Hoffmann-La Roche Ltd during the conduct of the study. GK, PD, GAK, CH, DA-S, NV and PF are shareholders in F. Hoffmann-La Roche Ltd. AD and NV were full-time employees of Roche Products Ltd during the conduct of the study. AD is currently employed at the MRC Clinical Trials Unit at UCL. MB and $\mathrm{RD}$ are full-time employees and shareholders in F. Hoffmann-La Roche Ltd and Genentech Inc. $\mathrm{CH}$ has an Alzheimer's disease-related patent planned which is relevant to this study.

Ethical standards: Institutional Review Boards (IRBs) approved the SCarlet RoAD and Marguertie RoAD studies, and all participants gave informed consent before participating.

Data sharing statement: Qualified researchers may request access to individual patient-level data through the clinical study data request platform: https://vivli. org. Further details on Roche's criteria for eligible studies are available here: https: / vivli.org/members/ourmembers. For further details on Roche's Global Policy on the Sharing of Clinical Information and how to request access to related clinical study documents, see here: https://www.roche.com/research and development/who_we_are_how_we_work/clinical_trials/our_commitment_to_ data_sharing.htm.

Open Access: This article is distributed under the terms of the Creative Commons Attribution 4.0 International License (http:/ / creativecommons.org/ licenses/by/4.0/), which permits use, duplication, adaptation, distribution and reproduction in any medium or format, as long as you give appropriate credit to the original author(s) and the source, provide a link to the Creative Commons license and indicate if changes were made.

\section{References}

1. Alzheimer's Association. 2019 Alzheimer's disease facts and figures Alzheimers Dement 2019; 15:321-387.

2. Wang S, Mims PN, Roman RJ, et al. Is beta-amyloid accumulation a cause or consequence of Alzheimer's disease? J Alzheimers Parkinsonism Dement 2016; 1:007.
3. Lee JC, Kim SJ, Hong S, et al. Diagnosis of Alzheimer's disease utilizing amyloid and tau as fluid biomarkers. Exp Mol Med 2019; 51:1-10.

4. Mucke L \& Selkoe D. Neurotoxicity of amyloid $\beta$-protein: Synaptic and network dysfunction. Cold Spring Harb Perspect Med 2012; 2:a006338.

5. Delbeuck X, Van der Linden M, Collette F. Alzheimer's disease as a disconnection syndrome? Neuropsychol Rev 2003; 13:79-92.

6. Ishii K. Amyloid positron emission tomography in the therapeutic strategies for Alzheimer's disease. Brain Nerve 2017; 69:809-818.

7. Klein G, Delmar P, Voyle N, et al. Gantenerumab reduces amyloid- $\beta$ plaques in patients with prodromal to moderate Alzheimer's disease: A PET substudy interim analysis. Alzheimers Res Ther 2019; 11:101.

8. Ostrowitzki S, Lasser RA, Dorflinger E, et al. A phase III randomized trial of gantenerumab in prodromal Alzheimer's disease. Alzheimers Res Ther 2017; 9.95.

9. Abi-Saab D, Andjelkovic M, Pross N, et al. MRI findings in the open label extension of the Marguerite RoAD study in patients with mild Alzheimer's disease. J Prev Alz Dis 2017; 4:339 (P336).

10. Fleisher AS, Chen K, Liu X, et al. Using positron emission tomography and florbetapir F18 to image cortical amyloid in patients with mild cognitive impairment or dementia due to Alzheimer disease. Arch Neurol 2011; 68:14041411.

11. Barthel H, Gertz HJ, Dresel S, et al. Cerebral amyloid- $\beta$ PET with florbetaben $(18 \mathrm{~F})$ in patients with Alzheimer's disease and healthy controls: A multicentre phase 2 diagnostic study. Lancet Neurol 2011; 10:424-435.

12. Klunk WE, Koeppe RA, Price JC, et al. The Centiloid Project: Standardizing quantitative amyloid plaque estimation by PET. Alzheimers Dement 2015; 11:1-15.e11-14.

13. Navitsky M, Joshi AD, Kennedy I, et al. Standardization of amyloid quantitation with florbetapir standardized uptake value ratios to the Centiloid scale. Alzheimers Dement 2018; 14:1565-1571.

14. von Rosenstiel P, Gheuens S, Chen T, et al. Aducanumab titration dosing regimen: 24-month analysis from PRIME, a randomized, double-blind, placebo-controlled Phase $1 \mathrm{~b}$ study in patients with prodromal or mild Alzheimer's disease. Neurology 2018; 90:Abstract S2.003.

15. Swanson C, Zhang Y, Dhadda S, et al. Treatment of early AD subjects with BAN2401, an anti-A $\beta$ protofibril monoclonal antibody, significantly clears amyloid plaque and reduces clinical decline. Alzheimers Dement 2018; 14 (Suppl):P1668. DT-1601-1607. 